\title{
Significance of Blood Glucose Control in Severe Nursing Care of ICU
}

\author{
Huang Huifang*, Lu Wanxian, Shan Miaohang \\ Intensive Care Unit, The First Affiliated Hospital of Jinan University, Guangzhou, China \\ Email address: \\ 183478928@qq.com(Huang Huifang),wanxian63@126.com(Lu Wanxian),105224073@qq.com(Shan Miaohang) \\ ${ }^{*}$ Corresponding author
}

\section{To cite this article:}

Huang Huifang, Lu Wanxian, Shan Miaohang. Significance of Blood Glucose Control in Severe Nursing Care of ICU. Journal of Surgery. Vol. 7, No. 6, 2019, pp. 180-183. doi: 10.11648/j.js.20190706.15

Received: September 21, 2019; Accepted: October 28, 2019; Published: November 9, 2019

\begin{abstract}
To investigate the effect of intensive blood glucose control in Intensive Care Unit (ICU) nursing and analyze its clinical significance. From August 2018 to August 2019, 94 severe patients treated with ICU in our hospital were divided into two groups according to the random digital table method. 47 cases in the control group received routine nursing, and 47 cases in the observation group were treated with blood glucose control and routine nursing management. The sequential organ failure score (SOFA), acute physiological and chronic health status score (APACHE II), hospitalization time in ICU and total hospitalization time were compared between the two groups. After nursing, the APACHE II score and SOFA score of the patients in the observation group were significantly lower than those in the control group $(\mathrm{P}<0.05)$; The total hospitalization time of ICU in the observation group was significantly shorter than that in the control group $(\mathrm{P}<0.05)$. Enhanced glycemic control in ICU intensive nursing can improve the clinical therapeutic effect and shorten the rehabilitation time. In addition, tight glycemic control can also optimize the prognosis and shorten hospital stay time. The intensive blood glucose control is worthy to be popularized in ICU.
\end{abstract}

Keywords: Blood Glucose Control, ICU, Intensive Care

\section{Introduction}

The severe condition of ICU patients is critical, which can cause serious stress reaction and metabolic disorder under the stimulation of the disease. Among them, abnormal glucose metabolism is more common, even in non-diabetic patients, it will also cause stress blood glucose elevation. It has been found that abnormal elevation of blood glucose is harmful to severe patients with ICU, which can cause a variety of complications and even lead to death of patients [1]. Some scholars pointed out that the level of blood glucose should be controlled between 4.1 and 6.1 $\mathrm{mmol} / \mathrm{L}$ to prevent adverse events. However, due to the low attention of some nurses to blood glucose control, the effect of blood glucose control is not ideal, which affects the therapeutic effect of the disease [2]. Therefore, this paper will strengthen blood glucose control in ICU intensive care, which is reported below.

\section{Data and Methods}

\subsection{General Information}

From August 2018 to August 2019, 94 severe patients were treated with ICU in our hospital. The random digital table method is used to divide it into two groups. There were 47 cases in the observation group (M 25, F 22, age $31 \geq 79$ years, mean $(61.38 \pm 6.30)$ years). Basic diseases were bronchial asthma (6 cases), pulmonary infection (12 cases), cerebrovascular disease ( 8 cases), severe trauma ( 8 cases), chronic obstructive pneumonia (7 cases) and heart failure (6 cases). There were 47 cases in the control group (M 27, F 20, age $33 \geq 77$ years, mean $(61.38 \pm 6.30)$ years). Basic diseases were bronchial asthma (5 cases), pulmonary infection (11 cases), cerebrovascular disease (9 cases), severe trauma ( 9 cases), chronic obstructive pneumonia (6 cases) and heart failure ( 7 cases). 


\subsection{Methods}

The patients in the control group received routine nursing care. Body temperature was observed and analyzed every $2 \mathrm{~h}$. Urine specific gravity, routine urine and ketone body were measured three times per day. Calories were accurately record to ensure the maintenance of normal body heat. Additionally, routine nursing care also includes: (1) condition evaluation: after admission, the general information, condition information and life index of the patient should be fully understood, and the condition of the patient should be preliminarily evaluated as the basis for carrying out nursing work; (2) Psychological intervention: after the sudden onset of the disease, the patient is often psychologically unbearable and the psychological burden is heavy. The patients should carry out psychological intervention and communicate with the patients. For the patients with unclear consciousness and serious illness, they should placate the patientsundefined emotions through limb movements, enhance their sense of security and improve the psychological status of the patients; (3) Catheter management: during the treatment of patients, it is necessary to retain a variety of pipelines, which should be properly managed to prevent accidental shedding and blockage of ducts, assess the risk of extubation of patients, and avoid related complications caused by improper extubation. Catheter is changed and cleaned ervery day and catheter-related infections (CRI) should be oberserved and stressed because of the high risk of infection of high-blodd-glucose patients; (4) Airway management: to give patients back tapping, sputum suction and other treatments to prevent airway obstruction, if necessary, negative pressure sputum aspiration should be given. Ensure the balance value of negative pressure during sputum aspiration, and find a comfortable position suitable for sputum aspiration of patients to prevent sputum asphyxia. (5) Life care: ICU patients need to stay in bed and maintain a position for a long time, which may lead to rashes and pressure sores in the pressure area. The nursing staff should change the position of the patient regularly. In addition, nursing staff should pay attention to keep the patient perineum clean.

On these basis above, the patients in the observation group were managed by blood glucose control, including: (1) Evaluation of blood glucose status: to understand the family history of diabetes, the history of own diabetes and the history of treatment, the patients with a history of diabetes should be given key nursing care; (2) Blood glucose monitoring: after entering ICU, microglucose meter should be used to detect blood glucose as soon as possible, mainly finger tip blood glucose, if it is more than $11.11 \mathrm{mmol} / \mathrm{L}$ as hyperglycemia, if it is less than $2.8 \mathrm{mmol} / \mathrm{L}$, hypoglycemia should be used as soon as possible, and the index of blood glucose should be detected by microglucose meter as soon as possible; The changes of blood glucose were monitored and treated according to the changes of blood glucose; The limitation of the use and the narrow detection range of the micrometer cannot meet the test of patients with hyperglycemia. If the patient's blood glucose is far below or over beyond the detection range of the micrometer, it is necessary to measure the blood glucose of venous blood further, so as not to affect the medical treatment due to the inaccurate detection of the patient's blood glucose. Once hyperglycemia occurs, the blood glucose level should be reported to the doctor immediately. (3) Blood glucose control: according to the patientundefineds blood sugar level to take different treatment measures, for patients with fasting blood glucose levels over $6.1 \mathrm{mmol} / \mathrm{L}$, should use insulin micropump, the initial pump injection speed of $1 \sim 2 \mathrm{U} / \mathrm{h}$; after below $6.1 \mathrm{mmol} / \mathrm{L}$, the treatment can be stopped. (4) Dietary guidance: guide patients to eat less sweet food, starches, high fat food, such as root vegetables, high sweet fruit or animal viscera. Instruct patients to take in more foods rich in vitamins but in low fat, so as to avoid unstable blood glucose caused by irregular diet and further cause adverse reactions in ICU. Maintain a diet obviously results in slow digestion and thus more stable blood glucose levels and greater satiety. After the blood glucose is maintained to a balanced state, it needs to be strengthened in the follow-up care and combined with food regulation to maintain the stability of blood glucose.

\subsection{Evaluation Criteria}

The sequential organ failure score (SOFA), acute physiological and chronic health status score (APACHE II), hospitalization time and total hospitalization time of ICU were compared between the two groups.

\subsection{Statistical Method}

SPSS21.0 software was used to process, $\overline{\mathrm{x}} \pm \mathrm{S}$ was used to represent the measurement data, $t$ test was used to indicate the difference $(\mathrm{P}<0.05)$, and there was significant difference between the two groups $(\mathrm{P}<0.05)$.

\section{Result}

\subsection{Comparison of SOFA Score and Apache II Score}

Table 1. The difference between SOFA score and Apache II score.

\begin{tabular}{|c|c|c|c|c|c|}
\hline \multirow{2}{*}{ Group } & \multirow{2}{*}{$\mathbf{N}$} & \multicolumn{2}{|l|}{ APACHE II } & \multicolumn{2}{|l|}{ SOFA } \\
\hline & & Before nursing care & After nursing care & Before nursing care & After nursing care \\
\hline Control group & 47 & $20.98 \pm 2.21$ & $17.28 \pm 2.27$ & $14.41 \pm 2.89$ & $9.55 \pm 0.49$ \\
\hline Observation group & 47 & $21.02 \pm 2.18$ & $13.08 \pm 2.14$ & $14.34 \pm 3.02$ & $7.57 \pm 0.67$ \\
\hline $\mathrm{t}$ & & 0.283 & 8.324 & 0.398 & 15.137 \\
\hline $\mathrm{P}$ & & 0.746 & 0.000 & 0.671 & 0.001 \\
\hline
\end{tabular}


Before nursing, the APACHE II score and SOFA score of the patients in the observation group were $(21.02 \pm 2.18)$ and (14.34 \pm 3.02$)$ respectively; The control group was $(20.98 \pm 2.21)$ and (14.41 \pm 2.89$)$, respectively, with no significant difference $(\mathrm{t} \leq 0.283, \geq 0.398, \mathrm{P}>0.05)$. After nursing, the APACHE II score and SOFA score of the patients in the observation group were $(13.08 \pm 2.14)$ and $(7.57 \pm 0.67)$ respectively; In the control group, the scores were (17.28 \pm 2.27$)$ and (9.55 \pm 0.49$)$, respectively, with significant difference $(\mathrm{t} \leq 8.324,15.137, \mathrm{P}<$ $0.05)$.

\subsection{Comparison of Hospitalization Time and Total Hospitalization Time of ICU}

The hospitalization time of ICU was $(5.25 \pm 1.18)$ days and the total hospitalization time was $(24.34 \pm 4.90)$ days in the observation group and $(7.60 \pm 1.23) \mathrm{d},(33.96 \pm 4.78)$ days in the control group, respectively, with significant difference $(\mathrm{t} \leq$ $7.245,7.550, \mathrm{P}<0.05)$.

Table 2. The difference of hospitalization time and total hospitalization time of ICU.

\begin{tabular}{llll}
\hline Group & N & Length of stay & Total length of stay \\
\hline Control group & 47 & $5.25 \pm 1.18$ & $24.34 \pm 4.90$ \\
Observation group & 47 & $7.60 \pm 1.23$ & $33.96 \pm 4.78$ \\
$\mathrm{t}$ & & 7.245 & 7.550 \\
$\mathrm{P}$ & & 0.001 & 0.000 \\
\hline
\end{tabular}

\section{Discussion}

Severe ICU patients are prone to stress hyperglycemia, which has a serious impact on their treatment, rehabilitation and life and health [3]. The mechanism of neuroendocrine system was analyzed, which was mainly due to the activation of neuroendocrine system under severe injury and stress, and the release of stress hormones, which led to the increase of muscle glycogen, liver glycogen decomposition, insulin resistance and blood glucose level [4]. It was found that hyperglycemia not only stimulates and aggravates inflammatory response, but also affects the function of various systems and organs, causes irreversible damage, increases the risk of infection, arrhythmia, myocardial infarction and death [5]. Therefore, in the nursing of severe patients, we should pay attention to blood glucose control and promote the recovery of the disease. It is the common pursuit of medical workers and researchers to control blood glucose and optimize the prevention and treatment effect of hyperglycemia in ICU ward. When hyperglycemia occurs, the body is prone to inflammatory reaction and irreversible damage to the heart, liver, kidney and other organs, and then increasing the risk of infection in patients and extending the treatment time. As of the weak immunity, the organ function of the patients in ICU often are also failing. Therefore, it is necessary to take measures to control patients' blood glucose in the nursing process.

In ICU patients, abnormal hormone regulation and the production of a large number of cytokines are potential reasons for the occurrence of stress hyperglycemia, and medical intervention and various iatrogenic factors will also increase the risk of stress hyperglycemia to some extent [6]. Most ICU patients will experience elevated blood glucose in the early stage. After entering the high metabolic stage, blood glucose level will decrease with the increase of energy consumption and gradually become normal. During high metabolic period, the increase of gluconeogenesis will lead to the decrease of glucose utilization by cells. Therefore, insulin resistance is enhanced, while ICU patients with severe illness tend to stay in bed for a long time due to their critical condition, and the insulin sensitivity of skeletal muscle is decreased [7]. Moreover, age may have a potential impact on hyperglycemia, and the occurrence of stress hyperglycemia will increase the incidence of poor prognosis of patients [8]. Actually, stress hyperglycemia is preventable. firstly, we can treat primary diseases, including effective infection control, maintain acid-base balance, which can reduce the release of stress hormones and blood glucose level. Regular blood glucose monitoring to master the duration and elevation of hyperglycemia. In addition, proper nutrition support is also important with adequate calories and proper distribution of nutrients [9].

In this study, after nursing, the APACHE II score and SOFA score of the patients in the observation group were significantly lower than those in the control group, and the total hospitalization time of the patients in the observation group was significantly shorter than that in the control group $(\mathrm{P}<0.05)$, suggesting that strengthening blood glucose control can improve the rehabilitation and prognosis of the patients. The investigation showed that the incidence of infection was $2.44 \%$ after strengthening blood glucose control, while the infection rate of routine nursing intervention was as high as $17.07 \%$, which indicated that blood glucose control could reduce the incidence of infection. When the blood glucose increased abnormally and fluctuated, the oxidative stress reaction of the patients increased, which could limit the normal signal transduction pathway of the cells and affect the function of endothelial cells, thus adversely affecting the life and health of the patients [10].

However, some meta analysis showed that tight glycemic control may lead to no mortality benefit to all critically ill adults [11]. Tomohide Yamada et al. employed network meta-analysis to examine the risk of mortality and hypoglycemia associated with different glycemic control targets in critically ill adults. They found that tight control did not reduce the risk of short-term mortality, but lead to severe hypoglycemia $(<2.2 \mathrm{mmol} / \mathrm{l})$ more frequently [12]. Preiser J C et al. recommended that a continuous glucose monitoring-based strategy may decrease the incidence and severity of hypoglycemia, every 5-15 min may be the best time interval [13]. Many clinical guidelines recommend target blood glucose between 140 and $180 \mathrm{mg} / \mathrm{dL}$ (7.8 and 10 $\mathrm{mmol} / \mathrm{L}$ ) for most patients in the intensive care unit (ICU) [14]. In our survey, we suggest the blood glucose between 4.1 and $6.1 \mathrm{mmol} / \mathrm{L}$ can reduce the adverse effect of blood glucose on the recovery of the disease and improve the prognosis. Actually, critical ill patients in the ICU from different clinical 
departments, including internal medicine and surgery, may suffer from a variety of diseases. Blood glucose targets in the ICU should be personalized based on assessment of preadmission glycemia [15].

To sum up, strengthening blood glucose control in ICU intensive nursing can improve the treatment effect and shorten the rehabilitation time, which is worth popularizing.

\section{References}

[1] Biolo G, Massolino B, Di F G, et al. Intensive insulin therapy increases glutathione synthesis rate in surgical ICU patients with stress hyperglycemia. [J]. Clinical Nutrition Supplements, 2018, 4 (2): 9-0.

[2] Chen Qi. Association of stress hyperglycemia and its control level with the prognosis of critically ill patients [J]. Journal of Bengbu Medical College, 2014, 39 (5): 587-589.

[3] Li Xin, Pan Huibin, Fu Kai, et al. Effect of standardized process management on blood glucose control and prognosis in patients with severe pneumonia mechanical ventilation $[\mathrm{J}]$. Clinical and Education of General Medicine, 2019, 17 (01): 33.

[4] Feng Zhanlian. Causes and nursing points of difficult control of blood glucose in elderly intensive care unit diabetic patients [J]. New World of Diabetes, 2019, 22 (02): 112-113.

[5] Yan-Bo L I, Yu-Hong L, Yue-Hai F. The necessary of heating device for enteral nutrition in ICU geriatric patients [J]. Clinical Journal of Medical Officers, 2016, 44 (3): 320-322.

[6] Li Li. Nursing Intervention on Stress Hyperglycemia in Critically Ill Patients with ICU. Guide of China Medicine, 2017, 15 (21): 260-261.
[7] Lesperance R N, Guillamondegui O D. Glycemic Control and Insulin Resistance [M]// Principles of Adult Surgical Critical Care. 2016.

[8] Chen Qunlin. Effects of different blood glucose control levels on prevention of infection and antibiotic efficacy in patients with intensive care [J]. Chinese Prescription Medicine, 2019, 17 (01): 115-116.

[9] Pan Yan. Blood glucose control and nursing care of ICU patients during enteral nutrition [J]. Electronic Journal of practical Clinical Nursing, 2018, 3 (13): 28 / 29.

[10] Mao Jiehua, Deng Daoxi, Zheng Xuyin. Nursing care of severe ICU patients with hyperglycemia controlled by continuous micropump intravenous insulin injection [J]. Jilin Medicine, 2017, 38 (05): 967 -969.

[11] Yatabe T, Inoue S, Sakaguchi M, et al. The optimal target for acute glycemic control in critically ill patients: a network meta-analysis [J]. Intensive Care Med 2017; 43: 16-28.

[12] Yamada T, Shojima N, Noma H, et al. Glycemic control, mortality, and hypoglycemia in critically ill patients: a systematic review and network meta-analysis of randomized controlled trials [J]. Intensive Care Medicine, 2017, 43 (1): $1-15$.

[13] Preiser J C, Lheureux O, Thooft A, et al. Near-Continuous Glucose Monitoring Makes Glycemic Control Safer in ICU Patients [J]. Critical Care Medicine, 2018, 46 (8): 1.

[14] Umpierrez G E, Pasquel F J. Management of Inpatient Hyperglycemia and Diabetes in Older Adults [J]. Diabetes Care, 2017, 40 (4): 509-517.

[15] Krinsley, Stephen J. The Long and Winding Road Toward Personalized Glycemic Control in the Critically Ill [J]. Journal of Diabetes Science and Technology, 2017, 12 (1): 26-32. 